\title{
Some Models for Contention Resolution in Cable Networks
}

\author{
Onno Boxma ${ }^{1}$, Dee Denteneer ${ }^{2}$, and Jacques Resing ${ }^{1}$ \\ 1 EURANDOM and Department of Mathematics and Computer Science \\ Eindhoven University of Technology \\ P.O. Box 513, 5600 MB Eindhoven, The Netherlands \\ \{Boxma, Resing\}@win.tue.nl \\ 2 Philips Research, Digital Signal Processing Group \\ Prof. Holstlaan 4, 5656 AA Eindhoven, The Netherlands
}

Dee.Denteneer@philips.com

\begin{abstract}
In this paper we consider some models for contention resolution in cable networks, in case the contention pertains to requests and is carried out by means of contention trees. More specifically, we study a number of variants of the standard machine repair model, that differ in the service order at the repair facility. Considered service orders are First Come First Served, Random Order of Service, and Gated Random Order of Service. For these variants, we study the sojourn time at the repair facility. In the case of the free access protocol for contention trees, the first two moments of the access delay in contention are accurately represented by those of the sojourn time at the repair facility under Random Order of Service. In the case of the blocked access protocol, Gated Random Order of Service is shown to be more appropriate.
\end{abstract}

\section{Introduction}

Cable networks are currently being upgraded to support bidirectional data transport, see e.g. van Driel et al. [1]. The system is thus extended with an 'upstream' channel to complement the 'downstream' channel that is already present. This upstream channel is time slotted and shared among many stations so that contention resolution is essential for upstream data transport. An efficient way to carry out the upstream data transport is via a request-grant mechanism, like in Digital Video Broadcasting [2]: stations request data slots in contention with other stations via contention trees. After a successful request, data transfer follows in reserved slots, not in contention with other stations.

A tractable model for the access delay due to this request procedure is an essential step toward a better understanding of such a request-grant mechanism, and expressions for the first moments of the distribution of the access delay are particularly relevant. However, the performance analysis of contention trees, see Mathys and Flajolet [3] or Tsybakov [4], has been carried out under the assumption of a Poisson source model. This does not easily lead to properties 
of the closed model for a finite number of stations that is appropriate when contention trees are used for reservation.

The machine repair model, also known as the computer terminal model or as the time sharing system (e.g. Kleinrock [5], Section 4.11; Bertsekas and Gallager [6], Example 3.22), is one of the key performance models that assumes a finite input population. Therefore, it is a promising model for contention resolution using contention trees. The basic model is as follows. There are $N$ machines working in parallel. After a working period a machine breaks down and joins the repair queue. At the repair facility, a single repairman repairs the machines according to some service discipline. Once repaired, a machine starts working again. In the basic model, the distribution of both the working time and the repair time of machines is assumed to be exponential and the service discipline at the repair facility is assumed to be First Come First Served (FCFS).

In this paper, we show that the machine repair model can be an appropriate model for contention resolution in cable networks for the case that so-called Capetanakis-Tsybakov contention trees are used for reservation (see [7/8]). It turns out that the average time spent in contention resolution, obtained via simulations, matches the average sojourn time at the repair facility in the basic machine repair model almost perfectly. However, the basic model fails to accurately predict the variance of the time spent in contention resolution.

Closer inspection of contention trees reveals a possible source for this mismatch. Contention trees, to be described in Section 2, deviate from queues with a FCFS discipline in that each station in a given group has the same probability of being served, irrespective of the instant at which it entered the group. This suggests that variants of the basic machine repair model are needed to obtain a more appropriate model for the time spent in contention resolution, and that these variants should have some randomness built into their service discipline. In this paper, we consider two such variants.

Firstly, we consider the machine repair model as described above with a random order of service (ROS) discipline. Here, after a repair, the next machine to be repaired is chosen randomly from the machines in the repair queue. We analyse the sojourn time distribution at the repair queue for this model by exploiting a close relationship with the machine repair model considered in Mitra [9], in which the service discipline at the repair facility is processor sharing (PS).

We shall see that the variance of the sojourn time under ROS gives an accurate prediction of the access delay of requests in contention, when the so-called free access protocol is used. However, the prediction is not accurate in case of the so-called blocked access protocol. For that protocol, we consider an extension of the machine repair model. In this extension, machines that broke down are first gathered in a waiting room before they are put in random order in the actual repair queue at the instants that this repair queue becomes empty. In the sequel this service discipline will be called gated random order of service (GROS). For the GROS service discipline, just as for the ROS discipline, the average sojourn time at the repair facility is identical to the average sojourn time in case of the FCFS discipline - which, as mentioned above, accurately matches the mean time 
spent in contention resolution. Hence, the emphasis of our analysis will be on obtaining an (approximate) expression for the variance of the sojourn time at the repair facility.

It is appropriate to comment briefly on the relevance of the variance of the access delay in contention resolution. Firstly, low variability implies low jitter. As such, access variability is a key performance measure in itself. However, the main reason for studying the variance of the access delay is that it is needed in understanding the total average waiting time in cable networks. This follows from the request grant mechanism employed, as explained in the first paragraph of this introduction. Data transfer in cable networks consists of two stages. In the first stage, bandwidth for data transfer is being requested via the contention procedure. Once successfully transmitted, the requests queue up in a second queue. In this queue, the service time distribution is given by the distribution of the number of packets for which transfer is being requested. Now, due to the phenomenon of request merging, which will be described in more detail in Section 2] the number of packets being requested depends on the time spent in contention so that the variance of the service time depends on the variance of the access delay in the contention resolution. Clearly, the variance of the service time is needed to estimate the average waiting time in this second queue. In the present paper we concentrate on the first stage; to analyze the overall delay is a topic for further study.

The rest of the paper is organised as follows. In Section 2 we describe the contention resolution process using contention trees in more detail. In Section 3 we review some of the properties of the basic machine repair model. Moreover, we derive expressions for the first two moments of the steady state sojourn time distribution. The machine repair model with ROS service discipline is considered in Section 4 Here, we first relate the model with ROS service discipline to the model with PS. After that, we briefly review the main results from Mitra 9] for the model with PS. In Section 5, we give an approximate derivation of the moments of the sojourn time in the model with GROS service discipline. In Section 6 we present numerical results which show that the models of Section 4 and 5 can be used to approximate the sojourn time for contention resolution in cable networks using contention trees.

\section{Access via Contention Trees}

Tree algorithms are a popular tool to provide access to a channel that is time slotted and shared among many stations. These algorithms and their many variants are also referred to as stack algorithms or splitting algorithms; we refer to Bertsekas and Gallager [6], Section 4.3, for a survey. In this paper, we will confine attention to the basic ternary tree, illustrated in Figure 1. The basic tree consists of nodes, and each of these nodes comprises three slots of the access channel. A collision occurs if more than one station attempts a transmission in a slot. These collisions are then resolved by recursively splitting the set of colliding stations, plus possible newcomers as explained below, into three disjoint subgroups. For 


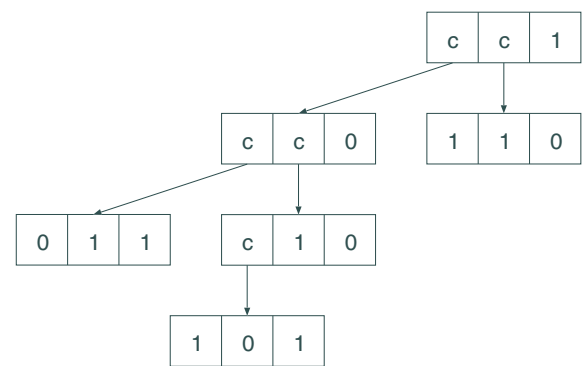

Fig. 1. Basic tree algorithm: slots of the tree with a collision (c) are recursively split until all slots are empty (0) or have a successful transmission (1)

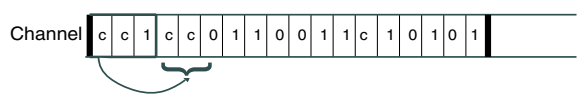

Fig. 2. Same tree as in Figure 11, with a breadth first ordering of the nodes

this, usually, a random mechanism is employed. This splitting continues until all tree slots are either empty or contain a successful transmission. This splitting process can be thought of as a tree, but takes place in time slots of the communication channel devoted to the contention resolution, so that the nodes of the tree must be time ordered. For this, we will use the breadth first ordering, as illustrated in Figure 2.

This basic tree algorithm must be complemented with a 'channel access protocol' that describes the procedure to be followed by stations that have data to transmit and that are not already contending in the tree. We consider two such access protocols: free access and blocked access. In the former protocol, access to the tree is free and any station can transmit a request in the next node of the tree, as soon as it has data to transmit. In the latter protocol, the tree is blocked so that new stations can only transmit requests in the root node of the tree that is started as soon as the current tree has been completed.

The stations exhibit the following behaviour:

- A station becomes active in the contention process upon generation of a data packet. In case of free access it will then transmit a request in the next tree node, randomly choosing one of the three slots in this node. In case of blocked access it will wait for the next new tree to be started and transmit its request in one of the slots of the root node of this tree.

- A station stays active until its request has been successfully transmitted.

- While active, the station can update its request (request merging). Hence, packets generated at such an active station do not cause extra requests.

- After successful transmission of the request, the station becomes inactive, to become active again upon the generation of a new data packet.

Note that request merging implies that the number of stations that can be active in contention is bounded. Exactly this property makes results on the performance of contention trees in open models, as investigated in e.g. Mathys and Flajolet [3] or Tsybakov [4], less relevant to contention resolution in cable 
networks. This property also explains the approach in this paper, which approximates the access delay in transmitting a request by means of the sojourn time in a machine repair model.

\section{Properties of the Basic Machine Repair Model}

First we introduce some notation and quote some properties of the basic machine repair model. The total number of machines in the system is denoted by $N$. The machines work in parallel and break down, independently, after an exponentially distributed working period with parameter $\lambda$. Machines that broke down queue up in the repair queue, where they are served FCFS by a single repairman. The repair times of machines are exponentially distributed with parameter $\mu$.

With the random variables $X$ and $Y$ we denote the steady state number of machines that are in $Q_{W}$ (i.e., are working) and that are in $Q_{R}$ (i.e., are in repair), respectively. Clearly, the number of working machines and the number of machines in repair evolve as Markov processes. Their steady state distributions are (in fact even for generally distributed working periods, cf. [5]10]):

$$
\operatorname{Pr}(X=k)=\operatorname{Pr}(Y=N-k)=\frac{\rho^{k} / k !}{\sum_{i=0}^{N} \rho^{i} / i !}, \quad k=0, \ldots, N,
$$

where $\rho:=\mu / \lambda$. For the mean and variance of $X$ and $Y$ we have

$$
\begin{aligned}
& \mathrm{E}(X)=\rho\left(1-B_{N}(\rho)\right), \quad \mathrm{E}(Y)=N-\mathrm{E}(X), \\
& \operatorname{var}(X)=\operatorname{var}(Y)=\mathrm{E}(X)-\rho B_{N}(\rho)[N-\mathrm{E}(X)],
\end{aligned}
$$

where $B_{N}(\rho)$ denotes Erlang's loss probability, which is given by

$$
B_{N}(\rho)=\frac{\rho^{N} / N !}{\sum_{i=0}^{N} \rho^{i} / i !}
$$

Indeed, it is well known that the number of operative machines has the same distribution as the number of busy lines in the classical Erlang loss model.

We now turn to the moments of the sojourn time of an arbitrary machine at the repair facility. To this end, consider the time epoch at which an arbitrary machine breaks down and jumps to the repair queue. Stochastic quantities related to this instant will be denoted by a subscript 1 . Thus $X_{1}$ is the number of working machines at this instant, and $Y_{1}$ is the number of machines in repair at this instant. From the arrival theorem, see Sevcik and Mitrani [11], it follows that the distributions of $X_{1}$ and $Y_{1}$ are given by (11), with $N$ replaced by $N-1$ :

$$
\operatorname{Pr}\left(X_{1}=k\right)=\operatorname{Pr}\left(Y_{1}=N-1-k\right)=\frac{\rho^{k} / k !}{\sum_{i=0}^{N-1} \rho^{i} / i !}, \quad k=0, \ldots, N-1
$$

The sojourn time of an arbitrary machine at the repair facility equals its own repair time plus the sum of the repair times of the machines already present at the repair facility. Thus, denoting this sojourn time by $S$, we have that 


$$
S=\sum_{i=1}^{Y_{1}+1} B_{i},
$$

with $B_{i}, i=1,2, \ldots$, a sequence of independent, exponentially distributed random variables with parameter $\mu$. Equation (6) enables us to obtain the LaplaceStieltjes transform (LST) of the sojourn time at the repair facility (see also 10]). Here, however, we are mainly interested in the first two moments of the sojourn time. These can be obtained by consideration of the moments of the random sum, i.e.,

$$
\begin{aligned}
\mathrm{E}\left(S_{F C F S}\right) & =\frac{1}{\mu}\left(N-\rho\left(1-B_{N-1}(\rho)\right)\right), \\
\operatorname{var}\left(S_{F C F S}\right) & =\frac{1}{\mu^{2}}\left(N-\rho B_{N-1}(\rho)\left[N-1-\rho\left(1-B_{N-1}(\rho)\right)\right]\right) .
\end{aligned}
$$

Now, for $N$ large and $N>>\mu / \lambda, B_{N}(\rho)$ goes to zero like $\rho^{N} / N$ !. Hence, in that case, the following are extremely sharp approximations:

$$
\mathrm{E}\left(S_{F C F S}\right) \approx \frac{N}{\mu}-\frac{1}{\lambda}, \quad \operatorname{var}\left(S_{F C F S}\right) \approx \frac{N}{\mu^{2}} .
$$

In Sections 4 and [5] shall study the sojourn time distribution at $Q_{R}$ under the assumption that the service discipline at that queue is Random Order of Service (ROS) and Gated Random Order of Service (GROS), respectively. The mean sojourn time in $Q_{R}$ is the same under FCFS, ROS and GROS; this is a direct consequence of Little's formula and the fact that the distribution of the number of customers in $Q_{R}$ is the same for any work-conserving service discipline that does not pay attention to the actual service requests of customers. We therefore focus in particular on the variance of the sojourn time in $Q_{R}$. Formula (9) shows that for the FCFS discipline, asymptotically, this variance is linear in the number of machines and does not depend on $\lambda$, the parameter of the distribution of the working times.

\section{The Model with ROS Service Discipline}

Again we consider the basic machine repair model, but now the service discipline at $Q_{R}$ is random order of service. For reasons that will soon become clear, we assume that the system contains not $N$ but $N+1$ machines. The main goals of this section are: (i) to determine the LST of the waiting time distribution at $Q_{R}$, (ii) to relate this distribution to the sojourn time distribution at $Q_{R}$ in case the service discipline is PS instead of ROS, and (iii) to determine the asymptotic behaviour of the variance of the waiting (and sojourn) time at $Q_{R}$ under the ROS discipline.

Consider a tagged machine, $C$, at the instant it arrives at $Q_{R}$. Let $S_{R O S}$ $\left(W_{R O S}\right)$ denote the steady state sojourn (waiting) time of $C$ at $Q_{R} . S_{R O S}$ is the sum of $W_{R O S}$ and a service time that is independent of $W_{R O S}$, and hence 
we can concentrate on $W_{R O S}$. We denote by $Y_{1}^{(N+1)}$ the number of machines in $Q_{R}$, as seen by $C$ upon arrival in $Q_{R}$. Introduce

$$
\phi_{j}(\omega):=\mathrm{E}\left[\mathrm{e}^{-\omega W_{R O S}} \mid Y_{1}^{(N+1)}=j+1\right], \quad \operatorname{Re} \omega \geq 0, \quad j=0, \ldots, N-1 .
$$

We can write, for $\operatorname{Re} \omega \geq 0$,

$$
\mathrm{E}\left[\mathrm{e}^{-\omega W_{R O S}} \mid W_{R O S}>0\right]=\sum_{j=0}^{N-1} \mathrm{P}\left(Y_{1}^{(N+1)}=j+1 \mid Y_{1}^{(N+1)}>0\right) \phi_{j}(\omega) .
$$

The following set of $N$ equations for the $N$ unknown functions $\phi_{0}(\omega), \ldots, \phi_{N-1}(\omega)$ holds:

$$
\begin{aligned}
\phi_{j}(\omega) & =\frac{\mu+(N-j-1) \lambda}{\mu+(N-j-1) \lambda+\omega}\left[\frac{(N-j-1) \lambda}{\mu+(N-j-1) \lambda} \phi_{j+1}(\omega)\right. \\
& \left.+\frac{\mu}{\mu+(N-j-1) \lambda}\left(\frac{1}{j+1}+\frac{j}{j+1} \phi_{j-1}(\omega)\right)\right] .
\end{aligned}
$$

Notice that the pre-factors of $\phi_{-1}(\omega)$ and $\phi_{N}(\omega)$ equal zero. Formula (11) can be understood in the following way. The pre-factor $(\mu+(N-j-1) \lambda) /(\mu+$ $(N-j-1) \lambda+\omega)$ is the LST of the time until the first 'event': Either an arrival at $Q_{R}$ or a departure from $Q_{R}$. An arrival occurs first with probability $(N-j-1) \lambda /(\mu+(N-j-1) \lambda)$. In this event, the memoryless property of the exponential working and repair times implies that the tagged machine $C$ sees the system as if it only now arrives at $Q_{R}$, meeting $j+2$ other machines there. A departure occurs first with probability $\mu /(\mu+(N-j-1) \lambda)$. In this event, $C$ is with probability $1 /(j+1)$ the one to leave the waiting room and enter the service position; if it does not leave, it sees $Q_{R}$ as if it only now arrives, meeting $j$ other machines there.

We can use (11) to obtain numerical values of $\mathrm{E}\left(W_{R O S} \mid W_{R O S}>0\right)$ and $\operatorname{var}\left(W_{R O S} \mid W_{R O S}>0\right)$. Formula (11) can also be used to study this mean and variance asymptotically, for $N \rightarrow \infty$. In fact, for this purpose we can also use the analysis given by Mitra [9] for a strongly related model: The machine-repair model with processor sharing at $Q_{R}$ and with $N$ (instead of $N+1$ ) machines. Denote the LST of the sojourn time distribution of a machine meeting $j$ machines at $Q_{R}$, in the case of processor sharing, by $\psi_{j}(\omega)$. A careful study of Formula (11) and the explanation following it reveals that exactly the same set of equations holds for $\psi_{j}(\omega)$, if in the PS case there are not $N+1$ but $N$ machines in the system. Not only do we have $\phi_{j}(\omega)=\psi_{j}(\omega), j=0, \ldots, N-1$, but it also follows from (5) that $\mathrm{P}\left(Y_{1}^{(N+1)}=j+1 \mid Y_{1}^{(N+1)}>0\right)=\mathrm{P}\left(Y_{1}^{(N)}=j\right), j=0, \ldots, N-1$. The above equalities, combined with (10), imply that $W_{R O S}$, conditionally upon it being positive, in the machine-repair system with $N+1$ machines, has the same distribution as the sojourn time under processor sharing in the corresponding system with $N$ machines. Adding a superscript $(N)$ for the case of a machinerepair system with $N$ machines, we can write:

$$
\mathrm{P}\left(S_{P S}^{(N)}>t\right)=\mathrm{P}\left(W_{R O S}^{(N+1)}>t \mid W_{R O S}^{(N+1)}>0\right) .
$$


This equivalence result between ROS and PS may be viewed as a special case of a more general result in [12] (see [13] for another special case).

Using (12), it is easily verified that, for the machine repair model with $N$ machines, $\mathrm{E} S_{R O S}=\mathrm{E} S_{P S}=\mathrm{E} S_{F C F S}$, just as indicated in Section 3 Multiplication by $t$ and integration over $t$ in (12) yields:

$$
\operatorname{var}\left(S_{P S}^{(N)}\right)=\frac{\operatorname{var}\left(W_{R O S}^{(N+1)}\right)}{\mathrm{P}\left(W_{R O S}^{(N+1)}>0\right)},
$$

where $\mathrm{P}\left(W_{R O S}^{(N+1)}>0\right)$ is easily obtained from (11). If $N$ is large and $N>\mu / \lambda$, then $\mathrm{P}\left(W_{R O S}^{(N+1)}=0\right)$ is negligibly small. The previous formula hence implies: For $N \rightarrow \infty, \operatorname{var}\left(S_{P S}^{(N)}\right) \sim \operatorname{var}\left(W_{R O S}^{(N)}\right)$ - and hence also $\operatorname{var}\left(S_{P S}^{(N)}\right) \sim \operatorname{var}\left(S_{R O S}^{(N)}\right)$.

For an asymptotic analysis of $\mathrm{E} W_{R O S}^{(N)}$ and $\operatorname{var}\left(W_{R O S}^{(N)}\right)$ we can thus immediately apply corresponding asymptotics of Mitra 9] for the PS-variant. Mitra [9] shows that $S_{P S}$ is hyper-exponentially distributed. This, in turn, immediately implies that (see Proposition 12 in [9]),

$$
\operatorname{var}\left(S_{P S}\right) \geq\left(\mathrm{E} S_{P S}\right)^{2}
$$

Hence $\operatorname{var}\left(S_{P S}\right)=\mathrm{O}\left(N^{2}\right)$ for $N \rightarrow \infty$, which sharply contrasts with the $\mathrm{O}(N)$ behavior for FCFS (cf. (9)).

\section{The Model with GROS Service Discipline}

In this section, we consider the model with GROS service discipline as described in Section 1 Again, we let $Y$ denote the number of machines in the total waiting area (i.e. waiting room plus waiting queue). Obviously the distribution of $Y$ equals the distribution of the number of machines in the repair queue in the standard model described in Section 3 and is given by (1).

We will now consider the sojourn time until repair, $S_{G R O S}$, of an arbitrary (tagged) machine for the model with GROS service discipline. Observe that

$$
S_{G R O S}=\sum_{i=1}^{Y_{1}^{(1)}} B_{i}^{(1)}+\sum_{i=1}^{Y_{1}^{(2)}+1} B_{i}^{(2)} .
$$

Here, the random variables $B_{i}^{(1)}$ and $B_{i}^{(2)}$ are independent, exponentially distributed service times with parameter $\mu$. The random variable $Y_{1}^{(1)}$ is the number of machines in the waiting queue (including the one in repair) at the instant that the tagged machine breaks down. The random variable $Y_{1}^{(2)}+1$ equals the random position allocated to the tagged machine in the waiting queue at the instant it is moved from the waiting room to the waiting queue.

This model is not a closed product-form network, so that an exact analysis of the sojourn time is considerably more difficult than the analysis for the models considered above. However, a particularly easy approximation of the first moments can be obtained, if one makes the following two assumptions: 
- The two components of $S_{G R O S}$ in (15) are uncorrelated.

- The random variables $Y_{1}^{(1)}$ and $Y_{1}^{(2)}$ are uniformly distributed on $0,1, \cdots, Y_{1}$, where the random variable $Y_{1}$ is as defined in Section 3 .

Neither assumption is strictly valid; however, for the case considered in which $N \lambda>\mu$ and $N$ large, they appear to be good approximations. Using (15) and the fact that $B_{N}(\rho) \rightarrow 0$ like $\rho^{N} / N$ ! for $N$ large and $N>>\mu / \lambda$, see above (9), it follows that

$$
\operatorname{var}\left(S_{G R O S}\right) \approx \frac{1}{\mu^{2}}\left[(N-\mu / \lambda)^{2} / 6+(4 N-2 \mu / \lambda) / 3\right] .
$$

Thus, for large $N$, the GROS variance is much larger than the variance in the machine repair model with the FCFS service discipline. However, it is considerably smaller than the variance in the machine repair model with the ROS service discipline.

\section{A Comparison}

We now turn to a comparison of the access delay due to contention resolution and the sojourn time in the variants of the machine repair model. In this comparison, we will confine ourselves to the first two moments of the various distributions: we consider first moments in Section 6.1 and standard deviations in Section 6.2

The procedures for contention resolution were described in Section 2, and the access delay due to contention resolution is the delay experienced by stations that use contention trees for reservation. More formally, it is defined as the number of tree slots elapsed from the instant a station becomes active until the instant its request is successfully transmitted. As already indicated in Section 2 there are no closed form expressions for the moments of the distribution of the access delay. Hence, these are obtained via simulation. In these simulations, the stations execute the procedure outlined in Section 2. Thus, we use a source model in which each of a finite number, $N$, of stations generates packets according to a Poisson process with rate $\lambda$, independently of the other stations.

The average delays thus obtained are denoted $\widehat{E S_{F}}$ and $\widehat{E S_{B}}$, for the 'free' and 'blocked' channel access protocol respectively. Likewise, the estimated standard deviations are denoted by $\widehat{\sigma_{F}}$ and $\widehat{\sigma_{B}}$. The 'hat' serves as a reminder that the moments are estimated from a simulation. We use 1000 trees in each simulation.

The moments of the sojourn time of the various machine repair models have been obtained in Sections 3 to 5 In utilizing the results from these sections, we will use $\mu=\log (3)$ for the rate of the service time distribution. The motivation behind this value is in Janssen and de Jong (14], Eq. 26-27). They show that the average number of nodes to complete a tree with $n$ contenders is well approximated by $n / \log (3)$. 
Table 1. Average access delay for reservation with free tree, $\widehat{E S_{F}}$, with blocked tree, $\widehat{E S_{B}}$, and expected sojourn time for the machine repair model, $E S$, for number of stations $N$, and total traffic intensity $\Lambda$

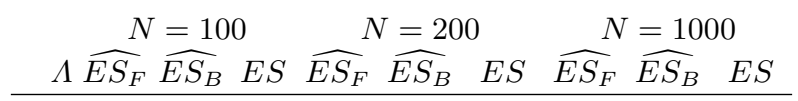

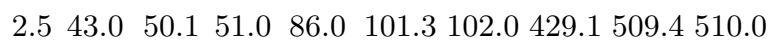

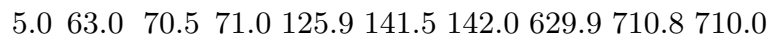

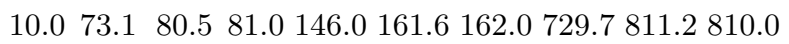

\begin{tabular}{llll}
$16.5 \quad 77.1 \quad 84.5$ & 84.9154 .5169 .5169 .9824 .9848 .5850 .0 \\
\hline
\end{tabular}

\subsection{First Moments}

The average access delays for the tree models and the expected sojourn time for the machine repair model are given in Table 1. There is only one entry in the table corresponding to the expected sojourn time, as it is the same for all variants of the machine repair model considered. In the table, we have varied the number of stations, $N$, and the total traffic intensity $\Lambda:=N \lambda$. The primary purpose of this table is to compare average access delay with expected sojourn time. Whence, the intensities are chosen so that $\Lambda$ is well above $\mu$, which is the case most relevant to access in cable networks.

From the figures we can draw various conclusions. Firstly, and most importantly, we observe that the expected sojourn time in the machine repair model provides an excellent approximation to the average access delay for reservation with contention trees. The agreement with the figures obtained via simulations with blocked access is almost perfect; the agreement with the results for free access is less good. The former result is closely related to a result in Denteneer and Pronk [15] on the average number of contenders in a contention tree.

Secondly, we see that free access is a more efficient access protocol than blocked access in that the average access delay with the former is smaller than the average delay with the latter. This result parallels the result for the open model and the Poisson source model, as graphically illustrated in Figure 16 of Mathys and Flajolet [3]. The considered variants of the machine repair model all lead to the same expected sojourn time and are apparently not sufficiently detailed as models to capture the first moment differences between the blocked and the free access protocols.

Finally, we observe that all quantities investigated in Table 1 depend approximately linearly on the number of stations (for the cases with $N>>\mu / \lambda$ ).

\subsection{Standard Deviations}

We next turn to a numerical comparison of the standard deviations in the various models. These are given in Table 2 again for different $N$ and $\Lambda$. 
Table 2. Standard deviations of the access delay for reservation with free tree, $\widehat{\sigma_{F}}$, with blocked tree, $\widehat{\sigma_{B}}$, and standard deviations for the basic machine repair model, $\sigma$, the ROS machine repair model, $\sigma_{R O S}$, and the GROS machine repair model, $\sigma_{G R O S}$ for number of stations $N$, and total traffic intensity $\Lambda$

\begin{tabular}{|c|c|c|c|c|c|c|c|c|c|}
\hline & & & $V=100$ & & & & $=1$ & 000 & \\
\hline & & & Reps & & $\operatorname{Tr}$ & & & Repai & \\
\hline$\Lambda$ & $\widehat{\sigma_{F}}$ & $\widehat{\sigma_{B}}$ & $\sigma \sigma_{R O S}$ & $\sigma_{G R O S}$ & $\widehat{\sigma_{F}}$ & $\widehat{\sigma_{B}}$ & $\sigma$ & $\sigma_{R O S}$ & $\sigma_{G R O S}$ \\
\hline 2.5 & 46.1 & 19.5 & 9.150 .45 & 22.8 & 429.1 & 85.1 & 28.8 & 509.64 & 210.4 \\
\hline 5.0 & 68.0 & 26.7 & 9.170 .18 & 30.6 & 629.9 & 261.6 & 28.8 & 709.39 & 291.7 \\
\hline 10.0 & 78.4 & 30.4 & 9.180 .13 & 34.6 & 729.7 & 299.2 & 28.8 & 809.34 & 332.4 \\
\hline 16.5 & 83.2 & 31.5 & 9.184 .06 & 36.2 & 786.6 & 310.6 & 28.8 & 848.73 & 348.4 \\
\hline
\end{tabular}

Several conclusions can be drawn from the table. Firstly, we observe that the standard deviation in either tree model changes with traffic intensity and grows approximately linearly with the number of stations. Neither of these properties is captured by the basic machine repair model; there, the standard deviation of the sojourn time is independent of the traffic intensity and grows only with the square root of the number of stations in the model.

Secondly, the standard deviation of the access delay in the blocked tree model corresponds closely to the corresponding figure for the GROS machine repair model. The difference between the two standard deviations is approximately $15 \%$. The results for the GROS model capture both the dependence on the traffic intensity and the dependence on the number of machines that is observed in the tree simulations. Similarly, the standard deviation of the access delay in the free tree model corresponds closely to the corresponding figure for the ROS machine repair model.

Looking more closely at the results, we see that the standard deviations obtained for the GROS machine repair model are always larger than those obtained in the blocked tree simulations. We consider this as a fundamental limitation of the machine repair model as an approximation. The batch nature of the contention trees implies that it takes some initial time before the first successful request is transmitted. After this initial period, successful transmissions occur fairly uniformly over the length of the trees. Thus the variability of the waiting period is somewhat reduced as compared to the proposed model in which the successful transmissions occur uniformly over the full length of the tree.

Thirdly, the standard deviations with the free access protocol far exceed those with the blocked access protocol. This result has no parallel in the open model. In fact, Figure 17 in Mathys and Flajolet [3] shows that the standard deviation of the delay with free access protocol is below the corresponding value with blocked access for most traffic intensities. However, for large traffic intensities just below the stability bound the order reverses and blocked access then results in smaller standard deviations. Of course, our simulations operate at total traffic intensities that exceed the stability bound for the open system. 
Summarizing, our numerical experiments show that the expected sojourn time in the repair stage perfectly matches the average access delay for both variants of the tree procedure. The sojourn time variance in the model with ROS service discipline gives a good approximation of the access delay variance when using free trees. Similarly, the sojourn time variance in the model with GROS service discipline gives a good approximation of the access delay variance when using blocked trees. More numerical results are presented in [16].

Acknowledgement. The authors like to thank Marko Boon for doing a major part of the numerical calculations.

\section{References}

1. Driel, C-J. van, van Grinsven, P.A.M., Pronk, V., Snijders, W.A.M.: The (r)evolution of access networks for the information super-highway. IEEE Communications Magazine 35 (1997) 2-10

2. Digital Video Broadcasting (DVB); DVB interaction channel for Cable TV distribution systems (CATV), working draft (Version 3), June 28, 2000, based on European Telecommunications Standard 300800 (March 1998)

3. Mathys, P., Flajolet, Ph.: Q-ary collision resolution algorithms in random-access systems with free or blocked channel access. IEEE Trans. Inf. Theory 31 (1985) 217-243

4. Tsybakov, B.: Survey of USSR contributions to random multiple-access communications. IEEE Trans. Inf. Theory 31 (1985) 143-165

5. Kleinrock, L.: Queueing Systems, Vol. 2. Wiley, New York (1976)

6. Bertsekas, D.P., Gallager, R.G.: Data Networks. Prentice-Hall, Englewood Cliffs, N.J (1992)

7. Capetanakis, J.I.: Tree algorithms for packet broadcast channels. IEEE Trans. Inf. Theory 25 (1979) 505-515

8. Tsybakov, B.S., Mikhailov, V.A.: Random multiple access of packets: Part and try algorithm. Probl. Peredachi Inf. 16 (1980) 65-79

9. Mitra, D.: Waiting time distributions for closed queueing network models of sharedprocessor systems. In: F.J. Kylstra (ed.), Performance'81, NHPC, Amsterdam (1981) 113-131

10. Kobayashi, H.: Modeling and Analysis. An Introduction to System Performance Evaluation Methodology. Addison-Wesley, Reading (Mass.) (1978)

11. Sevcik, K.C., Mitrani, I.: The distribution of queueing network states at input and output instants, In: M. Arato et al. (eds.), Performance'79, NHPC, Amsterdam (1979) 319-335

12. Borst, S.C., Boxma, O.J., Morrison, J.A., Núñez Queija, R.: The equivalence of processor sharing and service in random order. SPOR-Report 2002-01, Eindhoven University of Technology (2002)

13. Cohen, J.W.: On processor sharing and random order of service (Letter to the editor). J. Appl. Probab. 21 (1984) 937

14. Janssen, A.J.E.M., de Jong, M.J.M.: Analysis of contention tree-algorithms. IEEE Trans. Inf. Theory 46 (2000) 2163-2172

15. Denteneer, D., Pronk, V.: On the number of contenders in a contention tree. Proc. ITC Specialist Seminar, Girona (2001) 105-112

16. Boxma, O.J., Denteneer, D., Resing, J.A.C.: Some models for contention resolution in cable networks. EURANDOM Report 2001-037 (2001) 